
illusions above a carpet-like device by

SUBJECT AREAS:

TRANSFORMATION

OPTICS

METAMATERIALS

IMAGING AND SENSING

MICRO-OPTICS

Received

28 October 2014

Accepted

27 January 2015

Published

26 February 2015

Correspondence and requests for materials should be addressed to

G.P.W. lgpwang@

szu.edu.cn)

* These authors contributed equally to this work. \section{reflection holography}

\author{
Qiluan Cheng ${ }^{1 *}$, Kedi Wu ${ }^{2 *}$, Yile Shi ${ }^{3}$, Hui Wang ${ }^{3} \&$ Guo Ping Wang ${ }^{1,2}$
}

\begin{abstract}
${ }^{1}$ School of Physics and Technology, Wuhan University, Wuhan 430072, Hubei, P. R. China, ${ }^{2}$ College of Electronic Science and Technology, Shenzhen University, Shenzhen 518060, Guangdong, P. R. China, ${ }^{3}$ Institute of Information Optics, Zhejiang Normal University, Jinhua 321000, Zhejiang, P. R. China.
\end{abstract}

Realization of a perfect invisibility cloak still challenges the current fabricating technologies. Most experiments, if not all, are hence focused on carpet cloaks because of their relatively low requirements to material properties. Nevertheless, present invisibility carpets are used to hide beneath objects. Here, we report a carpet-like device to directionally conceal objects and further to create illusions above it. The device is fabricated through recording a reflection hologram of objects and is used to produce a time-reversed signal to compensate for the information of the objects and further to create light field of another object so as to realize both functions of hiding the objects and creating illusions, respectively. The carpet-like device can work for macroscopic objects at visible wavelength as the distance between objects and device is at decimeter scale. Our carpet-like device to realizing invisibility and creating illusions may provide a robust way for crucial applications of magic camouflaging and anti-detection etc.

$\mathrm{n}$ terms of transformation optics ${ }^{1,2}$ and conformal mapping ${ }^{3}$, different kinds of invisibility cloaks have been proposed, designed and demonstrated theoretically and experimentally. To cloak objects, a shell configuration $^{4-10}$ is designed to control electromagnetic waves to propagate around the enveloped objects while maintain original propagation wave front like passing through an equivalent free space. To overcome the limitation that objects to be hidden are generally enveloped by a cloak shell, complementary media -based distant cloaking devices are proposed and numerically demonstrated to hide an object outside the cloak shell ${ }^{11}$ and furthermore to create an optical illusion of changing one object into another object ${ }^{12,13}$. However, perfect invisibility cloaks require the constitutive materials with extreme properties such as anisotropy, inhomogeneity, singular dielectric parameters, or even negative index of refraction, which challenge the current fabricating technologies ${ }^{14,15}$, specifically in optical frequencies. So far, apart from a concept proof of metamaterial-based two-dimensional (2D) invisibility cloaks for hiding objects from millimeter-scale cylinder in the microwave region ${ }^{4}$ to living creatures with incoherent natural light ${ }^{8}$, as well as some testing experiments for creating illusions in microwave region ${ }^{13,16}$ and thermal heat ${ }^{17}$, most experiments, if not all, were focused on carpet cloaks-- a simplified model of shell cloaks, which can reshape the reflection light from a curve surface of the cloaks to make it looks like reflected from a plane mirror. As a result, when an object is placed beneath the curve surface of the carpet cloaks, it is unperceivable to the outside through reflection light ${ }^{18}$. Due to their relatively low requirements to material properties, different configurations of the carpet cloaks with $2 \mathrm{D}^{19-21}$ and three-dimensional (3D) ${ }^{22,23}$ structures by using manmade metal-contained metamaterials ${ }^{19}$ or metal-free all-dielectrics ${ }^{20-23}$ and even naturally available anisotropic materials $^{24,25}$ are constructed with working frequency ranging from microwave frequencies ${ }^{19}$ to Terahertz, nearinfrared and even visible $e^{23-25}$. The hidden objects also vary in size from wavelength scale ${ }^{19-23}$ to macroscopic $\mathrm{c}^{24,25}$. Nevertheless, all of the carpet cloaks are used to hide objects beneath the carpets (Fig. 1a).

Here, we experimentally demonstrate a kind of carpet-like devices to directionally hide an object above them (Fig. 1b) and further to create an illusion of transferring the above object to another one (Fig. 1c) ${ }^{26}$. The carpet-like devices are reflection holograms made of commercially available all-dielectric materials. By producing a timereversed signal to compensate for information of an object and further an additional light field of another object, the carpet-like devices can realize functions of hiding the object and creating illusion in the visible as the object is at centimeter scale in size and at a distance of several tens of centimeters away from the carpet-like devices. We believe these carpet-like devices to realizing invisibility and illusions should be more applicable in the fields such as magic camouflaging and anti-detection etc. 
a

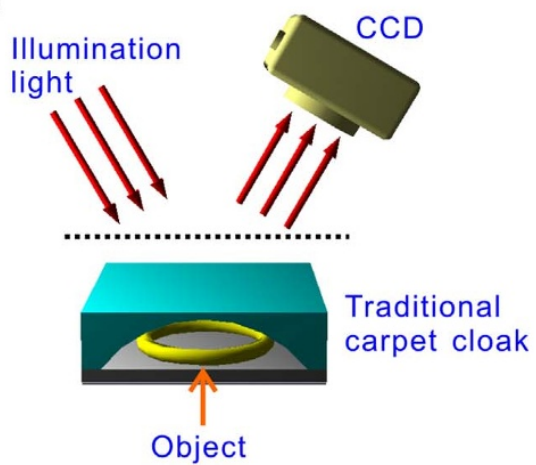

b

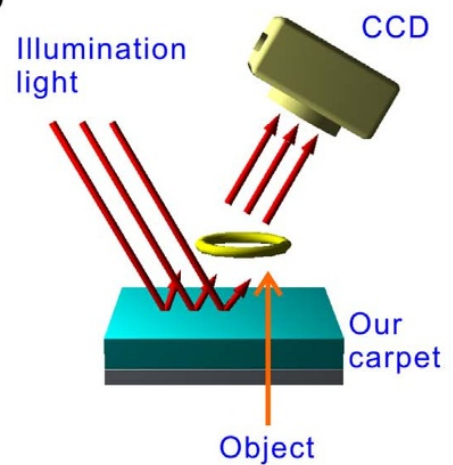

C

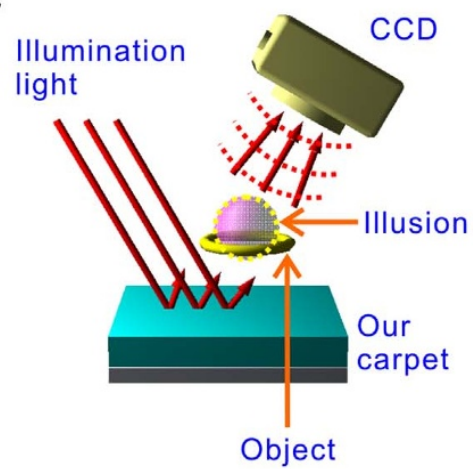

Figure 1 Working models of traditional carpet cloaks and our carpet-like devices. (a) A traditional carpet cloak is used to hide an object beneath. The light reflected by the under surface of the carpet cloak is distorted by the carpet layer so that the curved surface is detected as a plane mirror to the outside. Thus, objects placed in the cavity covered by the curved surface are invisible through reflection observation. (b) and (c), The present carpet-like devices are used to hide objects above them and create an illusion of transferring one object into another one. When illuminated by a light beam, the devices produces a time-reversed signal to compensate for the scattering effect of objects placed above the devices in the case of hiding the objects (b), and to further produce additional light field of another object simultaneously so as to make one object looking as another one by reflection observation (c).

\section{Results}

Theoretical basis and fabricating procedures of carpet-like devices. So far, time-reversal principle has been widely exploited to produce phase-conjugation signals for not only imaging beyond the diffraction limit ${ }^{27,28}$, focusing light into a scattering medium ${ }^{29-31}$, but also concealing objects and creating illusions ${ }^{32,33}$. Experimentally, the time-reversed light beam can be created through either linear ${ }^{29-33}$ or nonlinear optical effects ${ }^{34}$ such as holography and four-wave mixing. However, all the above applications are generally based upon transmission detection. Here, we employ reflection holography to produce reflection time-reversal light to realize hiding objects and creating illusions above a carpet-like device through reflection observation.
To construct a carpet-like device (C-1) for concealing object $\mathrm{O}_{1}$, we place $\mathrm{O}_{1}$ in plane $\mathrm{P} 1$ (Fig. 2) to record a reflection hologram of $\mathrm{O}_{1}$ in plane $\mathrm{P} 0$. While to get another carpet-like device (C-2) for creating optical illusions of changing object $\mathrm{O}_{1}$ to another object $\mathrm{O}_{2}$, we place objects $\mathrm{O}_{1}$ in plane $\mathrm{P} 1$ and $\mathrm{O}_{2}$ in plane $\mathrm{P} 2$ simultaneously in the recording procedure.

Experimentally, object $\mathrm{O}_{1}$ is a transparent two-heart picture embossed on a glass substrate, while object $\mathrm{O}_{2}$ is an amplitudemodulated smiling face logo (see Methods). Figures $3 \mathrm{a}$ and $3 \mathrm{c}$ show the schemes and actual photographs of two objects $\mathrm{O}_{1}$ and $\mathrm{O}_{2}$, and the left hand panels of Figs. $3 \mathrm{~b}$ and $3 \mathrm{~d}$ present the images of $\mathrm{O}_{1}$ and $\mathrm{O}_{2}$, respectively, as they are directly illuminated by a laser beam at $632.8 \mathrm{~nm}$. To quantitatively know the contrast of the images, we

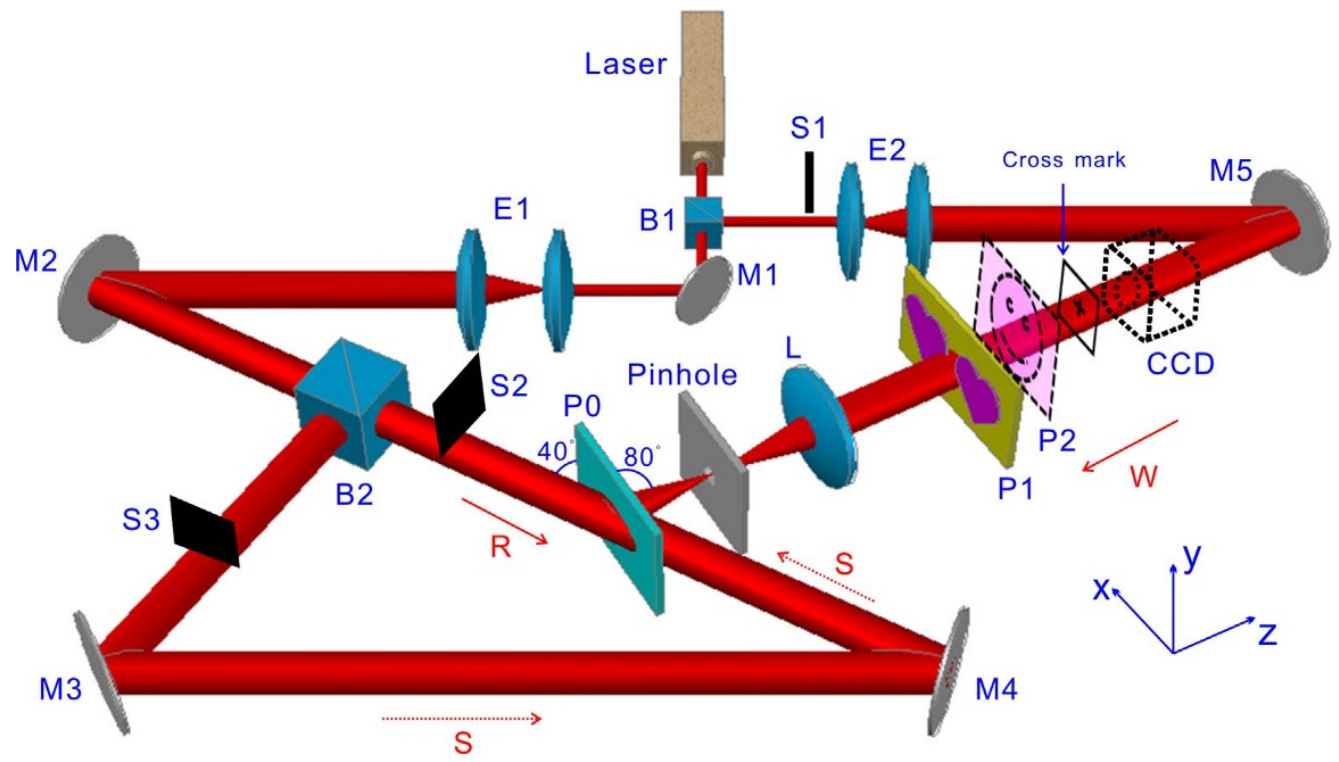

Figure $2 \mid$ Experimental setup for constructing and characterizing the carpet-like devices. A light beam with wavelength $\lambda=632.8 \mathrm{~nm}$ from a He-Ne laser is divided into three parts: object light $\mathrm{W}$, reference light $\mathrm{R}$ and reconstruction light $\mathrm{S}$ by beam splitters B1 and B2. M1-M5 are mirrors, E1 and E2 are beam expanders, S1, S2 and S3 are the shuttles, $\mathrm{L}$ is a lens with focal length $\mathrm{f}=10 \mathrm{~cm}, \mathrm{P} 0, \mathrm{P} 1$ and $\mathrm{P} 2$ are planes for recording plate, objects $\mathrm{O}_{1}$ and $\mathrm{O}_{2}$, respectively. The distance between object plane $\mathrm{P} 1$ and $\mathrm{L}$ is $20 \mathrm{~cm}$, which equals to the distance between $\mathrm{L}$ and $\mathrm{P} 0$. So P0 is the one-to-one image plane of $\mathrm{P} 1$. The pinhole is used to block the stray light. A charge-coupled device (CCD) placed $20 \mathrm{~cm}$ away behind $\mathrm{O}_{1}$ is used to take images of objects and monitor the characterization of carpet-like devices. A cross mark is placed between the CCD and object plane P1 as a reference mark for monitoring the effect of the carpet-like devices on other objects nearby $\mathrm{O}_{1}$. The $\mathrm{x}-\mathrm{y}$ plane of coordinate system is parallel to $\mathrm{P} 1$ and the $\mathrm{z}$ axis direction is anti-parallel to the light beam W. In exposure process, shuttles S1 and S2 are opened but S3 is closed, while in the process of characterizing the effects of finished carpet-like devices, shuttles $\mathrm{S} 1$ and $\mathrm{S} 2$ are closed but $\mathrm{S} 3$ is opened. 
a

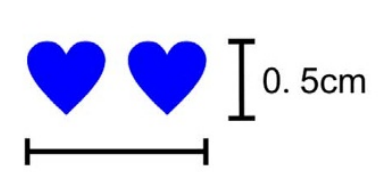

1. $3 \mathrm{~cm}$

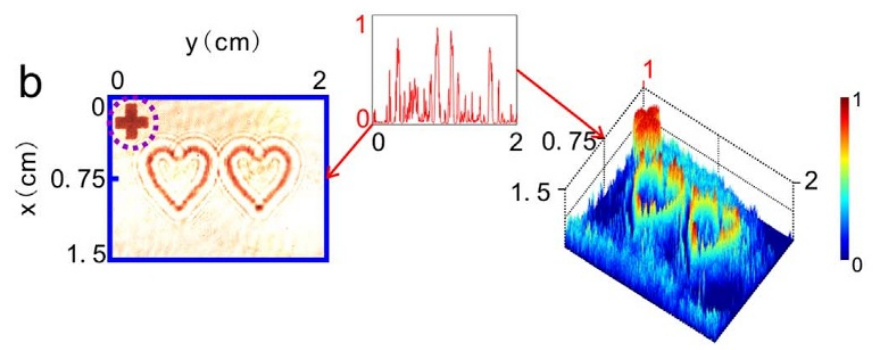

C

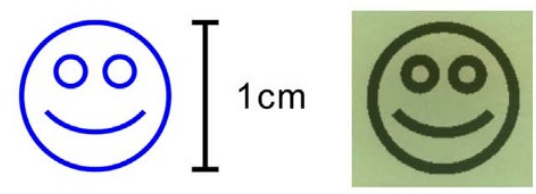

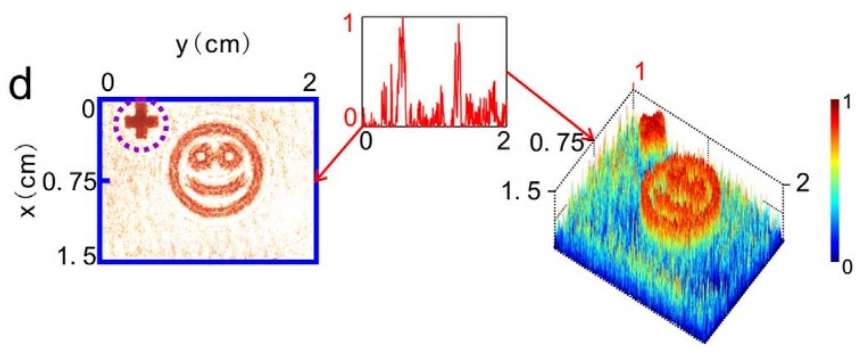

Figure $3 \mid$ Sketches of object $\mathrm{O}_{1}$ and object $\mathrm{O}_{2}$ used in the experiments. (a) Sketch of object $\mathrm{O}_{1}$ (left) and its actual photograph (right): a two-heart picture with area $1.3 \mathrm{~cm} \times 0.5 \mathrm{~cm}$. (b) Planar image of object $\mathrm{O}_{1}$ (left hand panel) received by CCD and its 3D intensity profile (right hand panel) when $\mathrm{O}_{1}$ is placed in $\mathrm{P} 1$ plane of the optical setup and is directly illuminated by a plane light. (c) Sketch of object $\mathrm{O}_{2}$ (left) and its actual photograph (right): a smiling-face logo with diameter $1 \mathrm{~cm}$. (d) Planar image of object $\mathrm{O}_{2}$ (left hand panel) received by CCD and its 3D intensity profile (right hand panel) when $\mathrm{O}_{2}$ is placed in $\mathrm{P} 2$ plane of the optical setup and is directly illuminated by a plane light. Insets of each picture are the line graphs of the corresponding images along the $y$ axis at $x=0.75 \mathrm{~cm}$.

show in the right hand panels of Figs. $3 \mathrm{~b}$ and $3 \mathrm{~d}$ their $3 \mathrm{D}$ profiles and the insets intensity line graphs of the images along the $y$ axis at $x=$ $0.75 \mathrm{~cm}$. We can see that in intensity line graph of $\mathrm{O}_{1}$, there are four feature peaks that correspond to the edges of two hearts, while in that of $\mathrm{O}_{2}$, two feature peaks correspond to the edges of smiling face. The dashed-line circled cross at the top left corner of the images is the image of a cross mark placed between object $\mathrm{O}_{1}$ and a charge coupled device (CCD) for reference.

After exposure, development, bleaching and drying, the carpetlike devices are finished (see Supplementary Table 2). Since the bleaching processing turns the composites of the recording medium into a dielectric material, the carpet-like devices obtained in our experiments are completely transparent.

Characterization of $\mathbf{C}-1$ in hiding object $\mathbf{O}_{1}$. To examine the effect of $\mathrm{C}-1$ in hiding object $\mathrm{O}_{1}$, we replace $\mathrm{C}-1$ in plane $\mathrm{P} 0$ and maintain the position of $\mathrm{O}_{1}$ in plane $\mathrm{P} 1$. Fig. $4 \mathrm{a}$ shows the planar image received by CCD when $\mathrm{C}-1$ is illuminated by reconstruction light $\mathrm{S}$ (left hand panel). From the figure we can hardly see any information of $\mathrm{O}_{1}$ except the background noise (caused by interference of parasitical light beams in the recording process). To get a quantitative description, we also show in the right hand panel the $3 \mathrm{D}$ intensity profile of the image and in the inset its line graph along the $y$ axis at $x=0.75 \mathrm{~cm}$. We find that, unlike the inset of Fig. 3b, there are no four feature peaks of $\mathrm{O}_{1}$ in the line graph except random back ground noise. It means that the time-reversed signal produced by $\mathrm{C}-1$ indeed compensates for the scattering effect of $\mathrm{O}_{1}$ and makes it invisible. A movie illustrating the process of concealing object $\mathrm{O}_{1}$ by using C-1 is displayed in Supplementary movie 1 .

In holographic conjugation technology, deviations of the replaced hologram from its original position and orientation etc. in the recording process may seriously destroy the function of the technology. We then check the effect of position and orientation deviations of carpet-like device $\mathrm{C}-1$ on hiding object $\mathrm{O}_{1}$ above. The left hand panel of Fig. $4 \mathrm{~b}$ shows the received planar image of CCD as C-1 is deviated transversally $10 \mu \mathrm{m}$ away from its original position along the $\mathrm{x}$ axis. The right hand panel and inset are the $3 \mathrm{D}$ intensity profile of the image and its line graph along the y axis at $x=0.75 \mathrm{~cm}$, respectively. We can see that there still exists no any information of $\mathrm{O}_{1}$. When the transversal deviation becomes as large as $650 \mu \mathrm{m}$ away from its original position in the $\mathrm{x}$ axis, however, the profile of two hearts turns to observable (Fig. 4c, left hand panel) and the feature peaks of $\mathrm{O}_{1}$ begins to become to readable (Fig. 4c, inset).

Figures $4 \mathrm{~d}$ and $4 \mathrm{e}$ exhibit the received planar images of CCD (left hand panels) and their corresponding intensity profiles (right hand panels) and line graphs along the $y$ axis at $x=0.75 \mathrm{~cm}$ (insets) as C-1 deviates longitudinally about $\pm 1 \mathrm{~mm}$ (approaching to or going away from the object) away from its original plane along the $\mathrm{z}$ axis, respectively. We find that such large longitudinal deviation is still tolerable for hiding object $\mathrm{O}_{1}$ : it is still hard to discriminate the feature peaks of object $\mathrm{O}_{1}$.

For the effect of orientation deviation of C-1 on hiding object $\mathrm{O}_{1}$, we find that a little deviation from its original orientation seriously destroys the result. Our experimental results show that when the orientation deviation of $\mathrm{C}-1$ is as small as $0.1^{\circ}$ away from its original orientation around, respectively, the y axis in the P0 plane (Fig. $4 \mathrm{f}$ ) or the $\mathrm{x}$ axis in the $\mathrm{P} 0$ plane (Fig. $4 \mathrm{~g}$ ), $\mathrm{O}_{1}$ becomes visible, indicating that a small rotation deviation from its original orientation destroys the concealing effect of $\mathrm{C}-1$. This is because, when the holographic plate is rotated by $0.1^{\circ}$ along the $\mathrm{x}(\mathrm{y})$ axis, the diffraction light beam will move along the $\mathrm{y}(\mathrm{x})$ axis of about $0.7 \mathrm{~mm}$ on the plane of lens $\mathrm{L}$, which is much larger than the tolerant deviation of the holographic plate to the transverse displacement deviation along the $\mathrm{x}(\mathrm{y})$ axis (about $650 \mu \mathrm{m}$ ).

It should be pointed out that, as mentioned before, any deviation of C-1from its original location may ruin its function of phaseconjugation signals. However, from above results we see that, in a certain range of displacement deviations occurring in the transversal and longitudinal directions, our carpet-like device still works well in hiding object. This can be attributed to the background noise of image in reconstruction.

Note that, although object $\mathrm{O}_{1}$ is concealed by $\mathrm{C}-1$, from Figs. $4 \mathrm{a}-$ $4 \mathrm{~g}$ we see that, no matter object $\mathrm{O}_{1}$ is concealed or not by $\mathrm{C}-1$, the cross mark placed between object $\mathrm{O}_{1}$ and CCD is always visible (dashed-line circled cross at the top left corner of each picture). This indicates that $\mathrm{C}-1$ just plays the role of concealing the object to be hidden but has no effect on other objects nearby.

Characterization of C-2 in transferring object $\mathrm{O}_{1}$ into $\mathrm{O}_{2}$. Figure 5a presents the planar image (left hand panel) taken by CCD when only object $\mathrm{O}_{1}$ is fixed in P1 plane (object $\mathrm{O}_{2}$ is removed) but $\mathrm{C}-2$ is replaced in $\mathrm{P} 0$ plane and is illuminated by 

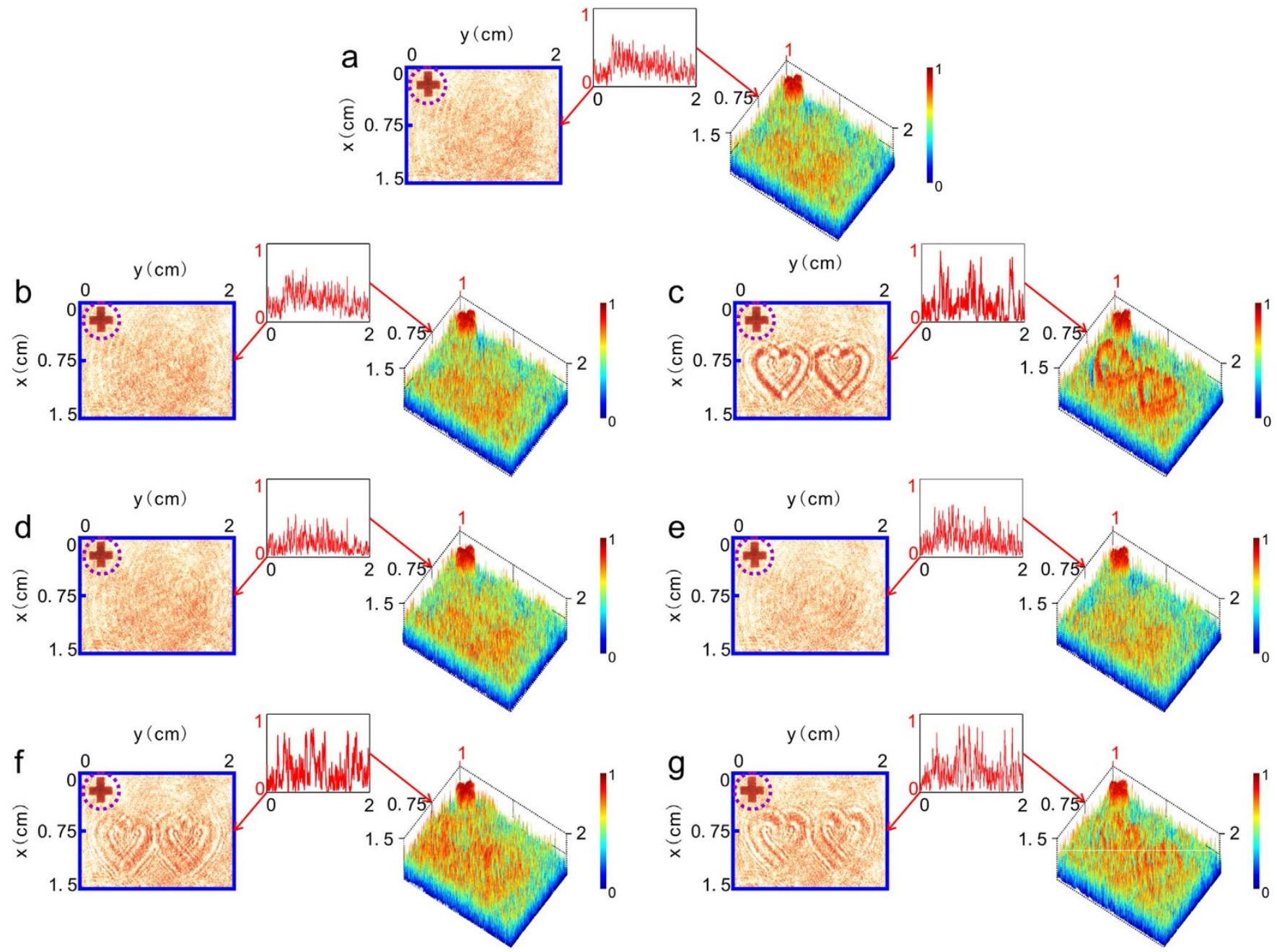

Figure $4 \mid$ Experimental results of hiding object $\mathrm{O}_{1}$. (a) Planar image (left hand panel) received by CCD and its 3D intensity profile (right hand panel) when $\mathrm{C}-1$ is precisely replaced in P0 plane of the optical setup. $(\mathrm{b}-\mathrm{c})$ Planar images (left hand panels) received by CCD and their corresponding 3D intensity profiles (right hand panels) as $\mathrm{C}-1$ is deviated transversally $10 \mu \mathrm{m}$ and $650 \mu \mathrm{m}$ away from its original position along the $\mathrm{x}$ axis, respectively. (d-e) Planar images (left hand panels) received by CCD and their corresponding 3D intensity profiles (right hand panels) as C-1 is deviated longitudinally about $\pm 1 \mathrm{~mm}$ (approaching to or going away from the object) away from its original plane along the $\mathrm{z}$ axis, respectively. (f-g) Planar images (left hand panels) received by CCD and their corresponding $3 \mathrm{D}$ intensity profiles (right hand panels) when orientation deviation of C- 1 is as small as $0.1^{\circ}$ away from its original orientation around, respectively, the $\mathrm{y}$ axis in the P0 plane (Fig. 4f) or the $\mathrm{x}$ axis in the P0 plane (Fig. 4g). Insets in each figures are the line graphs of the corresponding images along the $y$ axis at $x=0.75 \mathrm{~cm}$. Dashed-line circled cross at the top left corner of each picture is the image of the cross mark placed between object $\mathrm{O}_{1}$ and CCD.

reconstruction light $\mathrm{S}$. We see that, although we only place object $\mathrm{O}_{1}$ in plane $\mathrm{P} 1$, what is received by CCD camera is not the image of object $\mathrm{O}_{1}$ but instead an image of object $\mathrm{O}_{2}$ (smiling-face picture). From its 3D profile (right hand panel, Fig. 5a) and line graph of the image along the $y$ axis at $x=0.75 \mathrm{~cm}$ (inset, Fig. 5a), we see that not only there appear no the four feature peaks of object $\mathrm{O}_{1}$ (inset of Fig. 3b), but two feature peaks of $\mathrm{O}_{2}$ appear instead (inset of Fig. 3d). Consequently, we see that the carpet-like device C- 2 can not only produce a time-reversed signal for compensating for the scattered light fields of $\mathrm{O}_{1}$ but further create an additional light field of $\mathrm{O}_{2}$ so that $\mathrm{O}_{1}$ is looked as another different object, $\mathrm{O}_{2}$. A movie illustrating the process of creating an illusion of transferring $\mathrm{O}_{1}$ to $\mathrm{O}_{2}$ by using $\mathrm{C}$ 2 is displayed in Supplementary movie 2.

Figures 5b-5g exhibit the same as in Figs. 4b-4g, respectively, for checking the effect of position and orientation deviations of carpetlike device C-2 on creating optical illusions of transferring $\mathrm{O}_{1}$ to $\mathrm{O}_{2}$. Similar to concealing object $\mathrm{O}_{1}$ by $\mathrm{C}-1$, in a certain range of displacement deviations occurring in the transversal and longitudinal directions, C-2 also works well in creating an optical illusion. In addition, we also find, no matter object $\mathrm{O}_{1}$ is transferred into $\mathrm{O}_{2}$ or not by C-2, the cross mark placed between object $\mathrm{O}_{1}$ and CCD is always visible (dashed-line circled cross at the top left corner of each picture), meaning that C-2 also just plays the role of transferring object $\mathrm{O}_{1}$ into $\mathrm{O}_{2}$ but has no effect on other objects nearby $\mathrm{O}_{1}$.

All experimental results (Figs. 3-5) agree well with the numerical simulations based on computer generated holography ${ }^{35}$ (Supplementary Figs. 1-3), indicating that both numerical and experimental results verify that our approach to hiding objects and creating illusions above a carpet-like device is feasible.

\section{Discussions}

Recently, unidirectional invisibility devices based upon carpet cloaks $^{36}$ and parity-time symmetric structures ${ }^{37-41}$ are also inspiring some specific researching interests due to their relatively simplifying design and specific application potentials in wide fields such as directional military detection and biomedical imaging etc. For simplification, we only experimentally fabricate carpet-like devices that just work in one direction. However, our carpet-like devices can, in prin- 

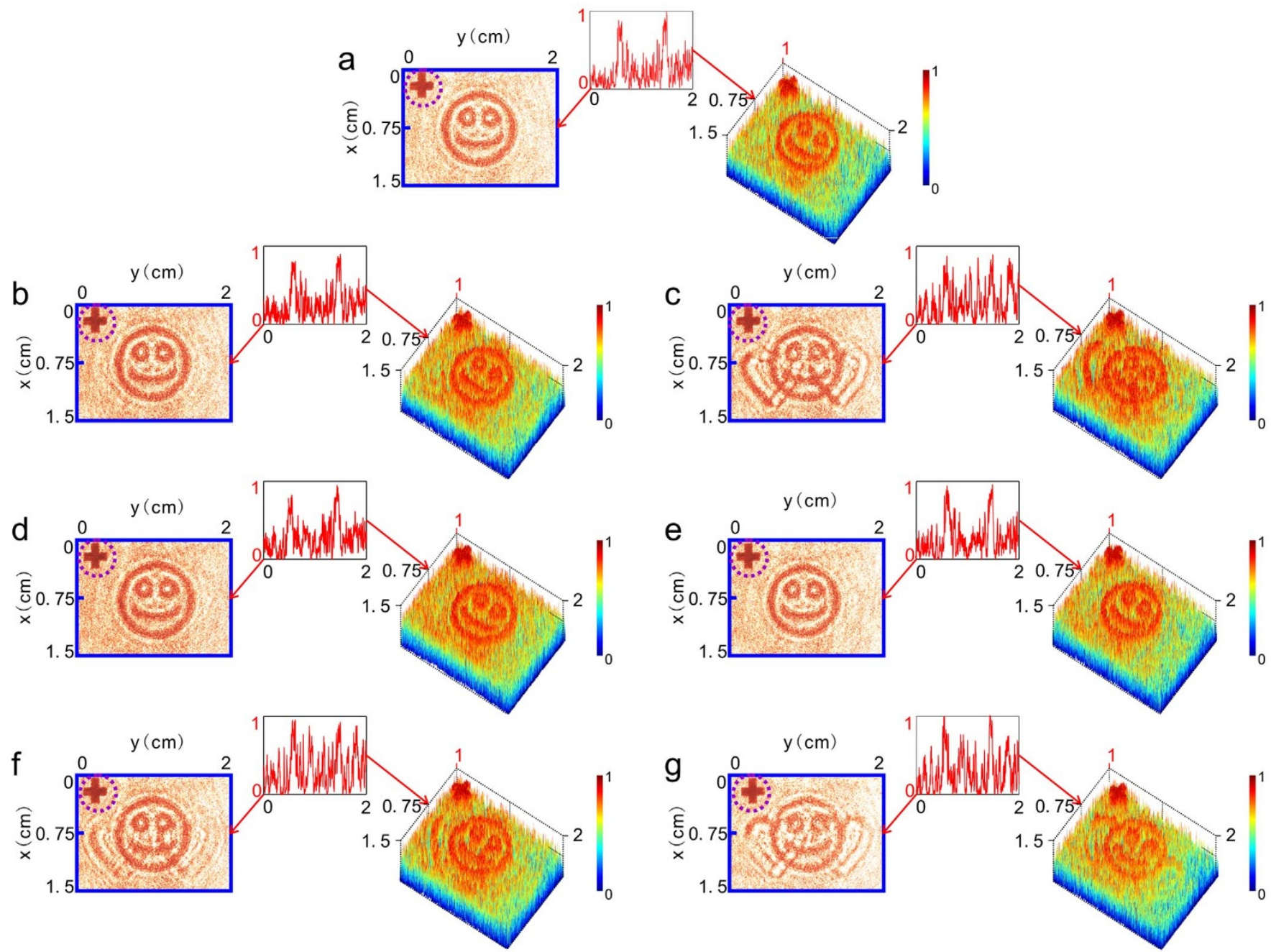

Figure 5 Experimental results of creating an illusion of transferring $\mathrm{O}_{1}$ into $\mathrm{O}_{2}$. (a-g) the same as in Figs. $4 \mathrm{a}-4 \mathrm{~g}$, respectively, for checking the effect of position and orientation deviations of carpet-like device C-2 on creating optical illusions of transferring $\mathrm{O}_{1}$ to $\mathrm{O}_{2}$.

ciple, be easily expanded to work at different directions by using multiple reference beams from different directions to construct the carpet-like devices, as we did in the cases of transmission observation $^{42}$. In addition, by using panchromatic holographic plates to construct the carpet-like devices, we can also make them working at multiple frequencies.

In conclusion, we have reported a kind of invisibility carpet-like devices to conceal objects and create illusions above them. The devices are experimentally realized by recording the reflection holograms of objects. When illuminated by a conjugated beam to the reference light in the recording procedure, the carpet-like devices can produce a time-reversed signal to conceal objects or further to create an additional light field so as to make one object looking as another one. The carpet-like devices can work for macroscopic objects (at centimeter scale) at visible wavelength when the distance between the objects and the carpet-like devices is at the decimeter scale. In addition, the devices are made of commercially available alldielectric materials. We believe that our work may open up a practical door to realizing invisibility and creating illusions and may inspire interesting applications in the fields such as magic camouflaging and anti-detection etc.

\section{Methods}

Objects $\mathrm{O}_{1}$ and $\mathrm{O}_{2}$ are both made of transmission holographic plates ${ }^{31}$. To fabricate $\mathrm{O}_{1}$, we mask a transparent film with two opaque heart-patterns printed on it on a holographic plate when exposing the plate by He-Ne laser with wavelength $\lambda=$
$632.8 \mathrm{~nm}$. After exposing, the plate is developed by D-19, bleached by mercuric chloride bleaching liquid and illuminated by a mercury vapor lamp in the dark room to change the refractive index profile of photosensitive materials on the plate. After fixed by F- 5 and bleached by R-10, the plate is fabricated to be the transparent object $\mathrm{O}_{1}$. (The formulas are detailed in Ref. 31.) To fabricate $\mathrm{O}_{2}$, a black film with a transparent smiling-face picture is masked on a holographic plate in the exposure process. Because $\mathrm{O}_{2}$ is an amplitude-modulated object, the two bleaching steps are not necessary in the post processing procedures, but all the other procedures maintain the same as those for creating $\mathrm{O}_{1}$.

The power of He-Ne laser is $60 \mathrm{~mW}$ before beam expanding. The carpet-like devices are moved by a step motor with $2.5 \mu \mathrm{m} / \mathrm{step}$ and rotated by a step motor with $0.1 \%$ step.

1. Ward, A. J. \& Pendry, J. B. Refraction and geometry in Maxwell's equations. J. Mod. Opt. 43, 773-793 (1996).

2. Pendry, J. B., Schurig, D. \& Smith, D. R. Controlling electromagnetic fields. Science 312, 1780-1782 (2006).

3. Leonhardt, U. Optical conformal mapping. Science 312, 1777-1780 (2006).

4. Schurig, D. et al. Metamaterial electromagnetic cloak at microwave frequencies. Science 314, 977-980 (2006).

5. Ruan, Z., Yan, M., Neff, C. W. \& Qiu, M. Ideal cylindrical cloak: perfect but sensitive to tiny perturbations. Phys. Rev. Lett. 99, 113903 (2007).

6. Cai, W., Chettiar, U. K., Kildishev, A. V. \& Shalaev, V. M. Optical cloaking with metamaterials. Nat. Photon. 1, 224-227 (2007).

7. Danner, A. J., Tyc, T. \& Leonhardt, U. Controlling birefringence in dielectrics. Nat. Photon. 5, 357-359 (2011).

8. Chen, H. et al. Ray-optics cloaking devices for large objects in incoherent natural light. Nat. Commun. 4, 2652 (2013).

9. Gömöry, F. et al. Experimental realization of a magnetic cloak. Science 335, 1466 (2012). 
10. Schittny, R., Kadic, M., Buckmann, T. \& Wegener, M. Invisibility cloaking in a diffusive light scattering medium. Science 345, 427-429 (2014).

11. Lai, Y., Chen, H. Y., Zhang, Z.-Q. \& Chan, C. T. Complementary media invisibility cloak that cloaks objects at a distance outside the cloaking shell. Phys. Rev. Lett. 102, 093901 (2009).

12. Lai, Y. et al. Illusion optics: the optical transformation of an object into another object. Phys. Rev. Lett. 102, 253902 (2009).

13. Li, C. et al. Experimental realization of a circuit-based broadband illusion-optics analogue. Phys. Rev. Lett. 105, 233906 (2010).

14. Pendry, J. B. \& Simth, D. R. Reversing light with negative refraction. Phys. Today 57, 37-43 (2004)

15. Chen, H., Chan, C. T. \& Sheng, P. Transformation optics and metamaterials. Nat. Mater. 9, 387-396 (2010).

16. Jiang, W., Qiu, C., Han, T., Zhang, S. \& Cui, T. Creation of ghost illusions using wave dynamics in metamaterials. Adv. Funct. Mater. 23, 4028-4034 (2013).

17. Han, T., Bai, X., Thong, J. T. L., Li, B. \& Qiu, C. Full control and manipulation of heat signatures: cloaking, camouflage and thermal metamaterials. Adv. Mater. 26, 1731-1734 (2014).

18. Li, J. \& Pendry, J. B. Hiding under the carpet: a new strategy for cloaking. Phys. Rev. Lett. 101, 203901 (2008).

19. Liu, R. et al. Broadband ground-plane cloak. Science 323, 366-369 (2009).

20. Valentine, J., Li, J., Zentgraf, T., Bartal, G. \& Zhang, X. An optical cloak made of dielectrics. Nat. Mater. 8, 568-571 (2009).

21. Gabrielli, L. H., Cardenas, J., Poitras, C. B. \& Lipson, M. Silicon nanostructure cloak operating at optical frequencies. Nat. Photon. 3, 461-463 (2009).

22. Ergin, T., Stenger, N., Brenner, P., Pendry, J. B. \& Wegener, M. Three-dimensional invisibility cloak at optical wavelengths. Science 328, 337-339 (2010).

23. Fischer, J., Ergin, T. \& Wegener, M. Three-dimensional polarization-independent visible-frequency carpet invisibility cloak. Opt. Lett. 36, 2059-2061 (2011).

24. Chen, X. et al. Macroscopic invisibility cloaking of visible light. Nat. Commun. 2 , 176 (2011).

25. Zhang, B., Luo, Y., Liu, X. \& Barbastathis, G. Macroscopic invisibility cloak for visible light. Phys. Rev. Lett. 106, 033901 (2011).

26. Wu, K. \& Wang, G. P. Hiding objects and creating illusions above a carpet filter using a Fourier optics approach. Opt. Express 18, 19894-19901 (2010).

27. Pendry, J. B. Time reversal and negative refraction. Science 322, 71-73 (2008)

28. Lerosey, G., De Rosny, J., Tourin, A. \& Fink, M. Focusing beyond the diffraction limit with far-field time reversal. Science 315, 1120-1122 (2007)

29. Xu, X., Liu, H. \& Wang, L. V. Time-reversed ultrasonically encoded optical focusing into scattering media. Nat. Photon. 5, 154-157 (2011).

30. Wang, Y., Judkewitz, B., DiMarzio, C. A. \& Yang, C. Deep-tissue focal fluorescence imaging with digitally time-reversed ultrasound-encoded light. Nat. Commun. 3, 928 (2012).

31. Judkewitz, B., Wang, Y., Horstmeyer, R., Mathy, A. \& Yang, C. Speckle-scale focusing in the diffusive regime with time reversal of variance-encoded light(TROVE). Nat. Photon. 7, 300-305 (2013).

32. Cheng, Q., Wu, K. \& Wang, G. P. All dielectric macroscopic cloaks for hiding objects and creating illusions at visible frequencies. Opt. Express 19, 23240-23248 (2011)
33. Cheng, Q., Wu, K., Shi, Y., Wang, H. \& Wang, G. P. Directionally hiding objects and creating illusions at visible wavelengths by holography. Sci. Rep. 3, 1974 (2013).

34. Longhi, S. Time-reversed optical parametric oscillation. Phys. Rev. Lett. 107, 033901 (2011).

35. Yu, Z. \& Jin, G. Computer Generated Holography (Tsinghua University Press, Beijing, 1984).

36. Landy, N. \& Smith, D. R. A full-parameter unidirectional metamaterial cloak for microwaves. Nat. Mater. 12, 25-28 (2012).

37. Lin, Z. et al. Unidirectional invisibility induced by PT-symmetric periodic structures. Phys. Rev. Lett. 106, 213901 (2011)

38. Longhi, S. Invisibility in PT-symmetric complex crystals. J. Phys. A 44, 485302 (2011).

39. Jones, H. F. Analytic results for a PT-symmetric optical structure. J. Phys. A 45, 135306 (2012).

40. Miri, M. A., Regensburger, A., Peschel, U. \& Christodoulides, D. N. Optical mesh lattices with PT symmetry. Phys. Rev. A 86, 023807 (2012).

41. Feng, L. et al. Experimental demonstration of a unidirectional reflectionless parity-time metamaterial at optical frequencies. Nat. Mater. 12, 108-113 (2013)

42. Goodman, J. W. Introduction to Fourier Optics (McGraw-Hill, New York, 2005).

\section{Acknowledgments}

This work is supported by the 973 Program (2011CB933600), the NSFC (Grant 11274247).

\section{Author contributions}

Q.L.C. performed the experiments, K.D.W. performed the theoretical analysis, Y.L.S assisted the experiments, H.W. and G.P.W. designed and conducted the experiments, G.P.W. conceived the idea and supervised the project. All authors contributed to the final version of the manuscript.

\section{Additional information}

Supplementary information accompanies this paper at http://www.nature.com/ scientificreports

Competing financial interests: The authors declare no competing financial interests.

How to cite this article: Cheng, Q., Wu, K., Shi, Y., Wang, H. \& Wang, G.P. Directionally hiding objects and creating illusions above a carpet-like device by reflection holography. Sci. Rep. 5, 8581; DOI:10.1038/srep08581 (2015).

This work is licensed under a Creative Commons Attribution 4.0 International License. The images or other third party material in this article are included in the article's Creative Commons license, unless indicated otherwise in the credit line; if the material is not included under the Creative Commons license, users will need to obtain permission from the license holder in order to reproduce the material. To view a copy of this license, visit http://creativecommons.org/licenses/by/4.0/ 